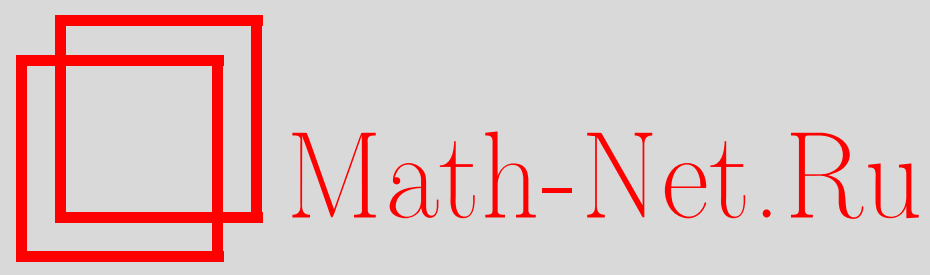

Е. В. Соколов, Замечание об отделимости подгрупп в классе конечных $\pi$-групп, Матем. заметки, 2003, том 73, выпуск 6, 904-909

DOI: https://doi.org/10.4213/mzm239

Использование Общероссийского математического портала Math-Net.Ru подразумевает, что вы прочитали и согласны с пользовательским соглашением http://www.mathnet.ru/rus/agreement

Параметры загрузки:

IP : 54.89 .56 .158

26 апреля 2023 г., 16:55:32

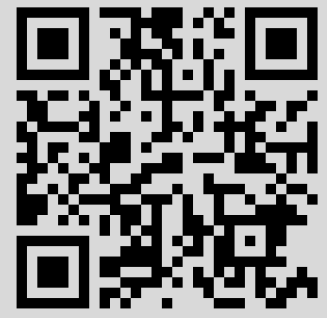




\title{
ЗАМЕЧАНИЕ ОБ ОТДЕЛИМОСТИ \\ ПОДГРУПП В КЛАССЕ КОНЕЧНЫХ $\pi$-ГРУПП
}

\section{Е.В. Соколов}

\begin{abstract}
В статье доказано, что если группа $G$ аппроксимируема в классе $\mathcal{N}$, то для каждой $\mathscr{N}$-подгруппы группы $G$ множество $\pi^{\prime}$-корней из этой подгруппы является $\pi$-отделимой $\mathcal{N}$-подгруппой.

Библиограффия: 5 названий.
\end{abstract}

1. Формулировка результатов. Пусть $\mathscr{K}$ - некоторьй класс групп. Напомним (см. [1]), что группа $X$ назьвается аппроксимируемой в классе $\mathscr{K}$ ( $\mathscr{K}$-аппроксимируeмой), если для любого неединичного элемента $g \in X$ существует гомоморфизм группы $X$ на некоторую группу из класса $\mathscr{K}$, переводящий $g$ в элемент, отличньй от 1 . Подгруппа $Y$ группы $X$ назьвается отделимой в классе $\mathscr{K}$ ( $\mathscr{K}$-отделимой), если для всякого элемента $g \in X \backslash Y$ можно указать такой гомоморфизм $\varphi$ группы $X$ нагруппу из $\mathscr{K}$, что $g \varphi \notin Y \varphi$.

Очевидно, что если все группы из класса $\mathscr{K}$ в свою очередь аппроксимируемы в некотором классе $\mathscr{L}$, то каждая $\mathscr{K}$-аппроксимируемая группа является $\mathscr{L}$-апроксимируемой. Очевидно также, что если $\mathscr{P}$ - гомоморфно замкнутьй класс групп и в произвольной группе из класса $\mathscr{K}$ все $\mathscr{P}$-подгрупшы (т.е. подгруппы, принадлежащие классу $\mathscr{P}$ ) являются $\mathscr{L}$-отделимыми, то из $\mathscr{K}$-отделимости всех $\mathscr{P}$-подгрупп произвольной группы $X$ следует их $\mathscr{L}$-отделимость. Именно на этом замечании основаны, например, доказательства большинства известных результатов о финитной отделимости всех конечно порожденных или всех циклических подгрупा свободного произведения с объединенной подгрупой, используюшие тот факт, что в свободном произведении с объединенной подгруппой двух конечных груп все конечно порожденные подгрупшы финитно отделимы.

Ситуация оказьвается более сложной, если $\mathscr{L}$-отделимость $\mathscr{P}$-подгрупп данной группы зависит от того, как подгруппы расположены в этой группе. Примером может служить отделимость в классе конечных $\pi$-групп ( $\pi$-отделимость).

Напомним, что если $\pi$ - некоторое множество простых чисел, то подгруппа $Y$ группы $X$ называется $\pi$-изолированной в $X$, если для всякого элемента $g \in X$ и для всякого простого числа $q \in \pi$ из $g^{q} \in Y$ следует, что $g \in Y$. Наименьшая $\pi$-изолированная подгруппа, содержащая подгруппу $Y$ группы $X$, назьвается $\pi$-изолятором $Y$ (об изоляторах в нильпотентных группах см., например, $[2, \S 4])$. Как обычно, $\pi^{\prime}$ обозначает множество всех простых чисел, не принадлежащих $\pi$. 
Легко видеть, что произвольная $\pi$-отделимая подгруппа должна быть $\pi^{\prime}$-изолированной. Очевидно также, что $\pi^{\prime}$-изолятор подгрупшы $Y$ содержит множество $\pi^{\prime}$-корней из $Y$, т.е. множество всех таких элементов, которые в некоторой $\pi^{\prime}$-степени лежат в $Y$ (мы будем обозначать его через $\sqrt[\pi^{\prime}]{Y}$ ).

Обратные утверждения, вообще говоря, не имеют места. Известно, однако, что для всякой подгрупшы $Y$ произвольной нильпотентной групшы множество $\sqrt[\pi^{\gamma}]{Y}$ является подгруппой (там же). Можно утверждать также, что в конечно порожденной нильпотентной группе без кручения подгрупша $\sqrt[\pi^{\prime}]{Y} \pi$-отделима (см. замечание в конце п. 3 ). Естественно возникает вопрос, справедливы ли аналогичные утверждения для подгруп произвольной группы, аппроксимируемой в классе $\mathcal{N}$ всех конечно порожденных нильпотентных групп без кручения. В этом направлении здесь будет доказано следующее утверждение.

TеоремА. Пусть группа $G$ аппроксимируема в классе $\mathcal{N}$. Тогда для каждой $\mathscr{N}$-подгруппь группь $G$ множсество $\pi^{\prime}$-корней из этой подгруппы является $\pi$-отделимой $\mathcal{N}$-подгруппой. $\quad B$ частности, кахдая $\pi^{\prime}$-изолированная $\mathcal{N}$-подгруппа группь $G$ т-отделима.

Заметим, что хотя формулировка теоремы не требует явно отделимости конечно порожденных нильпотентных подгрупп группы $G$ в классе $\mathcal{N}$, здесь это условие вьполняется автоматически (см. предложение 1 ниже). Приведем два утверждения, непосредственно вытекающих из сформулированной теоремы.

СлЕДСТВИЕ 1. Пусть группа $G$ является прямым произведением семейства свободных групп. Тогда для кажсдой конечно порохденной абелевой подгруппь группь $G$ множество $\pi^{\prime}$-корней является $\pi$-отделимой конечно порожденной абелевой подәруппой.

СлЕДСТВИЕ 2. Пусть $F-$ свободная группа, $w$ - әлемент группь $F$, порождающий в $F$ свой чентрализатор, $u G=\left\langle F * X ; w=x^{n}\right\rangle$-свободное произведение $c$ объединенной подәруппой группы $F$ и бесконечной ииклической группы $X$ с порохдающ,им $x, n \geqslant 1$. Тогда для каждой ииклической подгруппы группы $G$ множество $\pi^{\prime}$-корней является $\pi$-отделимой ииклической подгруппой.

Действительно, апшроксимируемость свободной группы в классе $\mathscr{N}$ хорошо известна, а для групшы $G$ из формулировки следствия 2 аналогичное утверждение доказано в работе [3].

Отметим также, что следствие 1 является обобщением известного результата о том, что всякая $\pi^{\prime}$-изолированная циклическая подгруппа свободной группы является $\pi$-отделимой (см., например, [4]).

2. Об отделимости подгрупп в классах групп без кручения. Аппроксимируемость в некотором классе групп (равносильная отделимости единичной подгруппы), вообще говоря, не влечет за собой отделимости даже циклических подгрупш. В этой связи представляет интерес следующее утверждение.

ПРЕДЛОЖЕНИЕ 1. Пусть $\mathscr{L}$ - класс әрупп без кручения, замкнутый относительно взятия подгрупп и прямых произведений конечного числа сомножителей, 
әруппа $G \mathscr{L}$-аппроксимируема, $H$ - произвольная поличиклическая подгруппа групnы G. Тогда

1) подгруппа $H$ L-отделима;

2) существует гомоморфизм әруппы $G$ на әруппу из класса $\mathscr{L}$, инбективный на nodzpynne $H$.

ДокАЗАтЕльство. Докажем сначала второе утверждение. Воспользуемся индукцией по рангу Гирша $r(H)$ подгруппы $H$, т.е. по числу бесконечных факторов в произвольном полициклическом ряде подгруппы $H$.

Так как группа $G$ без кручения, произвольная ееполициклическая подгруппа ранга 0 тривиальна, и утверждение в этом случае очевидно.

Пусть теперь $r(H)>0$. Обозначим через $\Omega$ семейство всех нормальных подгрупш группы $G$, фактор-группы по которым принадлежат классу $\mathscr{L}$. Ввиду условий, наложенных на класс $\mathscr{L}$, пересечение конечного числа подгрупп из $\Omega$ снова принадлежит $\Omega$.

Пусть $h$ - некоторый элемент подгрупшы $H$, отличный от 1, и $N \in \Omega$ - подгруппа, не содержашая $h$. Положим $H_{1}=H \cap N$. Тогда группа $H / H_{1}$ содержит элемент бесконечного порядка $h H_{1}$ и, следовательно, $r\left(H_{1}\right)<r(H)$. В силу индуктивного предположения найдется подгруппа $N_{1} \in \Omega$ такая, что $H_{1} \cap N_{1}=1$. Отсюда $H \cap\left(N \cap N_{1}\right)=H_{1} \cap N_{1}=1$.

Доказательство отделимости будем вести индукцией по минимальной длине полициклического ряда подгруппы $H$.

В случае, когда $H=1$, искомое утверждение следует из $\mathscr{L}$-аппроксимируемости группы $G$. Поэтому будем считать, что $H$ является расширением своей нормальной $\mathscr{L}$-отделимой подгрупшы $H_{1}$ при помощи циклической групшы.

Предположим, что существует такой элемент $g \in G \backslash H$, что для всякой подгруппы $M \in \Omega$ вьполнено $g \in H M$.

Выберем подгруппу $N \in \Omega$ таким образом, чтобы $H \cap N=1$ и $g \notin H_{1} N$. Пусть также $h$ - порождаюший подгруппы $H$ по модулю $H_{1}$. Тогда для каждой подгруппы $M \in \Omega$ вьполнено $h^{-m(M)} g \in H_{1} M$ для некоторого целого числа $m(M)$, отличного от нуля.

Если подгруша $M \in \Omega$ лежит в $N$, то $h^{m(M)-m(N)} \in H_{1} N$, но $H \cap N=1$, поэтому $h^{m(M)-m(N)} \in H_{1}$ и $h^{-m(N)} g \in H_{1} M$. Поскольку подгрупша $H_{1} \mathscr{L}$-отделима, отсюда следует, что $h^{-m(N)} g \in H_{1}$ и $g \in H$. Получаем противоречие с выбором элемента $g$. Предложение 1 доказано полностью.

3. Изоляторы в группах, аппроксимируемых конечными $\pi$-группами. Будем говорить, что порядок элемента $g$ группы $X$ по модулю некоторой подгруппы $Y$ конечен и равен $n$, если $g^{n} \in Y$ и $g^{m} \notin Y$ для каждого $m$ такого, что $0<m<n$.

ПРЕДЛОЖЕНИЕ 2. Пусть группа $X$ аппроксимируема конечными $\pi$-группами, $Y$ - нильпотентная подгруппа группы $X$ ступени с. Тогда мнохество $\sqrt[\pi^{\prime}]{Y}$ является нильпотентной подгруппой группы $X$ ступени с. Если подгруппа $Y$ конечно порожсдена и порядки всех элементов из $\sqrt[\pi^{\prime}]{Y}$ по модулю подгруппы $Y$ ограничены в совокупности, то и подгруппа $\sqrt[\pi^{\prime}]{Y}$ является конечно порохденной.

ДокАЗАТЕЛЬСТво. Будучи апроксимируемой конечньми $\pi$-группами, группа $X$ вкладывается в декартово произведение семейства конечных $\pi$-групा $X_{i}, i \in I$. Обозначим через $Y_{i}$ проекцию подгруппы $Y$ на группу $X_{i}$. 
Очевидно, что проекция $\pi^{\prime}$-изолятора $U$ подгрупшы $Y$ на каждую групшу $X_{i}$ совпадает с $Y_{i}$. Поэтому подгруппа $U$ вкладьвается в декартово произведение нильпотентных групп $Y_{i}$, каждая из которых имеет ступень не вьше $c$, и, следовательно, сама является нильпотентной группой ступени $c$. Отсюда сразу же следует, что $U=\sqrt[\pi^{\prime}]{Y}[2]$.

Предположим теперь, что подгрупп $Y$ конечно порождена и порядки всех элементов из $U$ по модулю подгрупшы $Y$ ограничены в совокупности числом $q$ (представляющим собой, очевидно, $\pi^{\prime}$-число), но при этом подгруппа $U$ не является конечно порожденной. Тогда найдется фактор $\xi_{i+1}(U) / \xi_{i}(U)$ верхнего центрального ряда групшы $U$, не являющийся конечно порожденной группой.

Ввиду отсутствия $\pi^{\prime}$-кручения в групе $X$ подгруппа $\xi_{i}(U) \pi^{\prime}$-изолирована в $U$ и фактор-группа $U / \xi_{i}(U)$ - это группа с однозначным извлечением $\pi^{\prime}$-корней [2, лемма 4.7]. Поэтому отображение подгрупшы $\xi_{i+1}(U) / \xi_{i}(U)$, переводящее элемент $x$ в $x^{q}$, является вложением этой подгрушы в конечно порожденную нильпотентную грушу $Y / \xi_{i}(U) \cap Y$. Полученное противоречие доказьвает наше утверждение.

Приведем одно простое следствие предложения 2.

СлЕДСТВИЕ. Пусть $X$ - некоторая группа, $\pi$ - произвольное непустое множество простых чисел, $1 \leqslant c<\infty$, и пусть для любого числа $p \in \pi$ выполнень следующие условия:

1) группа $X$ аппроксимируема конечными р-группами;

2) все $p^{\prime}$-изолированные нильпотентные подгруппы группы $X$ ступени не выше с р-отделимы.

Тогда все $\pi^{\prime}$-изолированные нильпотентные подгруппь группь $X$ ступени не выше с т-отделимы.

ДокАЗАТЕЛЬСтво. Действительно, пусть $Y-\pi^{\prime}$-изолированная нильпотентная подгруппа группы $X$ ступени не выше $c, g \in X$ - произвольный элемент, не принадлежащий $Y$. Из предложения 2 следует, что для любого числа $p \in \pi$ множество $\sqrt[p^{\prime}]{Y}$ является нильпотентной подгруппой групшы $X, p^{\prime}$-изолированной, а значит, и $p$-отделимой. Если порядок $g$ по модулю подгрупшы $Y$ конечен и равен $n$, то для любого простого делителя $p$ числа $n$ выполнено $g \notin \sqrt[p^{\prime}]{Y}$. Если же порядок $g$ по модулю подгруппы $Y$ бесконечен, то $g \notin \sqrt[p^{\prime}]{Y}$ для каждого числа $p \in \pi$.

Заметим, что в конечно порожденной нильпотентной группе без кручения для любого простого числа $p$ каждая $p^{\prime}$-изолированная подгруппа $p$-отделима [5]. Последнее утверждение позволяет распространить этот результат на случай произвольного множества простых чисел $\pi$. Впрочем, это можно сделать и непосредственно, модифицировав соответствующее рассуждение из [5].

4. Доказательство теоремы. Используя предложение 2, мы докажем утверждение, несколько более общее, чем то, что было сформулировано в теореме.

ПРЕДЛОЖЕНИЕ 3. Пусть $\mathscr{K ~ - ~ к л а с с ~ ә р у п п ~ б е з ~ к р у ч е н и я , ~ а п п р о к с и м и р у е м ы х ~ к о - ~}$ нечными т-группами, замкнутый относительно взятия подгрупп и прямых произведений конечного числа сомножителей, и пусть в любой группе из ЖК для каждой $\mathcal{N}$-подгруппы ступени не выше $c, 1 \leqslant c<\infty$, множество $\pi^{\prime}$-корней является $\pi$-отделимой $\mathscr{N}$-подгруппой. Если группа $G \quad \mathscr{K}$-аппроксимируема, то и в $G$ 
для кахдой $\mathscr{N}$-подгруппь ступени не выше с мнохество $\pi^{\prime}$-корней является п-отделимой $\mathcal{N}$-подгруппой.

Пусть снова $\Omega$ обозначает семейство всех нормальных подгрупп грушшы $G$, факторгруппы по которьм принадлежат классу $\mathscr{K}, H$ - конечно порожденная нильпотентная подгруппа групшы $G$ ступени не выше $c$. В соответствии с условием для каждой подгруппы $M \in \Omega$ множество $\sqrt[\pi^{\prime}]{H M / M}$ является конечно порожденной нильпотентной $\pi$-отделимой подгруппой группы $G / M$. Отсюда следует, в частности, что подгруппа $H M / M$ имеет конечный индекс в $\sqrt[\pi^{\prime}]{H M / M}[2]$.

В силу предложения 1 существует такая подгруппа $N \in \Omega$, что $H \cap N=1$. Пусть также $k=\left[\sqrt[\pi^{\prime}]{H N / N}: H N / N\right], q$ есть произведение всех $\pi^{\prime}$-чисел, не превосходяших $k$. Покажем, что для каждой подгруппы $M \in \Omega$, лежащей в $N$, порядки элементов подгруппы $\sqrt[\pi^{\prime}]{H M / M}$ по модулю $H M / M$ не превосходят $k$.

Рассмотрим сначала произвольньй элемент $h \in H$, порождающий в $H \pi^{\prime}$-изолированную подгруппу. Из условия легко следует, что для каждой подгруппы $M \in \Omega$ $\pi^{\prime}$-изолятор подгруппы $\langle h M\rangle$ в групп $G / M$ снова будет циклической подгруппой.

Пусть $\langle u N\rangle-\pi^{\prime}$-изолятор в групше $G / N$ подгруппы $\langle h N\rangle$. Тогда для некоторого $\pi^{\prime}$-числа $n$ вьполнено равенство $(u N)^{n}=h N$, причем без ограничения общности можно считать, что $n>0$. Так как $H \cap N=1$, подгруппа $\langle h N\rangle \pi^{\prime}$-изолирована в $H N / N$, и потому число $n$ является порядком $u N$ по модулю подгруппы $H N / N$.

Если далее подгруппа $M \in \Omega$ лежит в $N$ и $\langle v M\rangle-\pi^{\prime}$-изолятор подгруппы $\langle h M\rangle$ в группе $G / M,(v M)^{m}=h M$, то $(v N)^{m}=h N$ и, так как подгруппа $\langle u N\rangle \pi^{\prime}$-изолирована в $G / N, v N \in\langle u N\rangle$. Поскольку группа $G / N$ без кручения, отсюда следует, что $m \mid n$.

Пусть теперь $x M$ - произвольньй элемент подгруппы $\sqrt[\pi^{\prime}]{H M / M}$, и пусть для некоторого $\pi^{\prime}$-числа $r$ вьполнено $(x M)^{r} \in H M / M$. Пусть также $\langle h M\rangle-\pi^{\prime}$-изолятор подгрупшы $\left\langle(x M)^{r}\right\rangle$ в подгрупе $H M / M,\langle v M\rangle-\pi^{\prime}$-изолятор подгрупшы $\langle h M\rangle$ в группе $G / M$.

Так как $H \cap M=1$, подгруппа $\langle h\rangle \pi^{\prime}$-изолирована в $H$, и в силу доказанного вьше $[\langle v M\rangle:\langle h M\rangle] \leqslant k$. Очевидно далее, что $x M \in\langle v M\rangle$, поэтому порядок элемента $x M$ по модулю подгрупшы $\langle h M\rangle \leqslant H M / M$ также не превосходит $k$.

Обозначим через $\Sigma$ семейство всех нормальных подгрупп конечного $\pi$-индекса группы $G$ и положим $K=\bigcap_{L \in \Sigma} H L$. Легко видеть, что подгрупа $K$ является $\pi$-отделимой.

Пусть $g$ - произвольньй элемент из $K$. Тогда $g \varphi \in H \varphi$ при любом гомоморфизме $\varphi$ группы $G$ на конечную $\pi$-групшу и, следовательно, $g M \in \sqrt[\pi^{\prime}]{H M / M}$ для всякой подгруппы $M \in \Omega$. Из доказанного выше вытекает, что если $M$ лежит в $N$, то $g^{q} \in H M$. Заметим теперь, что по предложению 1 подгруппа $H \quad \mathscr{K}$-отделима, поэтому $g^{q} \in H$ и $g \in \sqrt[\pi^{\prime}]{H}$. Обратное включение $\sqrt[\pi^{\prime}]{H} \subseteq K$ очевидно.

Таким образом, для любой $\mathcal{N}$-подгрушы $H$ ступени не вьше $c$ множество $\sqrt[\pi^{\prime}]{H}$ является $\pi$-отделимой подгруппой в $G$. В частности, каждая $\pi^{\prime}$-изолированная $\mathcal{N}$-подгруппа группы $G$ ступени не вьше $c \pi$-отделима. Как было только что показано, порядки элементов $\sqrt[\pi^{\prime}]{H}$ по модулю подгрупшы $H$ ограничены в совокупности. Из предложения 2 теперь следует, что множество $\sqrt[\pi^{\prime}]{H}$ является конечно порожденной нильпотентной подгруппой в $G$. Предложение 3 доказано.

Автор выражает благодарность профессору Д. И. Молдаванскому за ряд ценных советов и замечаний при написании этой статьи. 


\section{СПИСОК ЦИТИРОВАННОЙ ЛИТЕРАТУРЫ}

[1] Мальцев А.И. О гомоморфизмах на конечные группы // Ученые записки Иван. гос. пед. ин-та. 1958. Т. 18. С. 49-60.

[2] Холл $\Phi$. Нильпотентные группы // Математика. Периодический сборник переводов иностранных статей. № 1. М.: Мир, 1968. С. 3-36.

[3] Baumslag G. On the residual nilpotence of certain one-relator groups // Comm. Pure Appl. Math. 1968. V. 21. № 5. P. 491-506.

[4] Азаров Д. Н. О нильпотентной аппроксимируемости свободных произведений свободных групп с циклическим объединением // Матем. заметки. 1998. Т. 64. №1. С. 3-8.

[5] Логинова Е. Д. Финитная аппроксимируемость свободного произведения двух групп с коммутирующими подгруппами // Сиб. матем. ж. 1999. Т. 40. № 2. С. 395-407.

Ивановский государственный университет

Поступило

E-mail: ev-sokolov@yandex.ru

15.11.2001

Исправленный вариант

16.07.2002 